\title{
A modified stochastic Gompertz model for tumour cell growth
}

\author{
C.F. Lo* \\ Department of Physics, Institute of Theoretical Physics, The Chinese University of Hong Kong, \\ Hong Kong SAR, PR China
}

(Received 4 June 2008; final version received 9 September 2008)

\begin{abstract}
Based upon the deterministic Gompertz law of cell growth, we have proposed a stochastic model of tumour cell growth, in which the size of the tumour cells is bounded. The model takes account of both cell fission (which is an 'action at a distance' effect) and mortality too. Accordingly, the density function of the size of the tumour cells obeys a functional Fokker-Planck Equation (FPE) associated with the bounded stochastic process. We apply the Lie-algebraic method to derive the exact analytical solution via an iterative approach. It is found that the density function exhibits an interesting kink-like structure generated by cell fission as time evolves.
\end{abstract}

Keywords: bounded random process; Fokker-Planck equation; Lie-algebraic method

Cancer is a major death cause in our modern society. In recent years increasing attention has been paid to the investigation of tumour growth because a better understanding of the highly complex process is of paramount importance for the development of more successful treatment strategies $[8,12]$. Taking the advantage of the methods of physics and engineering, most studies stem out of mechanistic population growth models which consist of one or more differential equations [17]. Despite their simplicity, such models of tumour growth make possible the description of the principal regularities and provide effective guidelines for cancer therapy, drug development and clinical decision-making [19]. In the past two decades, the deterministic Gompertz law of population growth has been widely used to describe in vivo tumour growth in experimental oncology [2,5,10,18,20,22]. If $x(t)$ is the size of the tumour cell at time $t$, then the Gompertz law models the cell growth by the equation

$$
\frac{\mathrm{d} x}{\mathrm{~d} t}=A_{1} x-A_{2} x \ln x, \quad A_{2}>0,
$$

where $A_{1}$, the intrinsic growth rate of the tumour cell, is a parameter related to the initial mitosis rate and $A_{2}$, the growth deceleration factor, is related to the antiangiogenic processes. However, it should be stressed that quite often discrepancies exist between clinical data and theoretical predictions, due to more or less intense environmental fluctuations. For instance [9]] analysed the effect of distinct chemotherapeutic strategies for the growth of avascular tumours, and confirmed that an environment like chemotherapy would affect tumour growth behaviour and lead to morphological transitions under certain conditions. Therefore, a better model is needed to reflect the external randomness that affects the cell growth behaviour.

A few years ago, Ref. [8] proposed a stochastic version of the Gompertz law to account for random fluctuations of the model parameters. They assume that the growth

\footnotetext{
*Email: cflo@phy.cuhk.edu.hk 
deceleration factor $A_{2}$ does not change, while the variability of environmental conditions induces fluctuations in the intrinsic growth rate $A_{1}$. By assuming that the intrinsic growth rate varies in time according to

$$
\theta(t)=A_{1}+\sigma \varepsilon(t)
$$

where $A_{1}$ is the constant mean value of $\theta(t), \sigma>0$ is the diffusion coefficient, and $\varepsilon(t)$ is a Gaussian white noise process, the proposed stochastic model is defined by the stochastic differential equation

$$
\mathrm{d} x=\left\{A_{1} x-A_{2} x \ln x\right\} \mathrm{d} t+\sigma x \mathrm{~d} z_{t},
$$

where $\mathrm{d} z_{t}$ denotes the standard Wiener process. By Ito's lemma Equation (3) implies that the exponent $\psi \equiv-\ln x$ follows the Ornstein-Uhlenbeck process [11]:

$$
\mathrm{d} \psi=\left\{\left(\frac{1}{2} \sigma^{2}-A_{1}\right)-A_{2} \psi\right\} \mathrm{d} t+\sigma \mathrm{d} z_{t}
$$

with the long term mean $\left((1 / 2) \sigma^{2}-A_{1}\right) / A_{2}$. This model has been applied to simulate the effects of a time-dependent therapy for the case of a parathyroid tumour [1].

Recently, Ref. [15] generalised the stochastic Gompertz model of tumour cell growth to include both cell fission and mortality at given rates, and formulated the extended model in terms of a functional Fokker-Planck Equation (FPE) of the form ${ }^{1}$

$$
\begin{aligned}
\frac{\partial P(x, t)}{\partial t}= & \frac{\partial^{2}}{\partial x^{2}}\{D(x, t) P(x, t)\}-\frac{\partial}{\partial x}\{g(x, t) P(x, t)\}+\alpha^{2} B(\alpha x, t) P(\alpha x, t)-(B(\alpha x, t) \\
& +\mu(x, t)) P(x, t),
\end{aligned}
$$

where $P(x, t)$ denotes the density function of cells of size $x \geq 0$ at time $t \geq 0, D(x, t) \equiv$ $D_{0}(t) x^{2}$ is the dispersion coefficient, $g(x, t) \equiv\left[g_{0}(t)-g_{1}(t) \ln x\right] x$ is the rate of growth, $\mu(x, t) \equiv \mu_{0}(t)$ is the rate of death, and $B(\alpha x, t) \equiv B_{0}(t)$ is the rate at which cells divide into $\alpha$ equally sized daughter cells. Here $\alpha>1$ is regarded as a constant, and the functions $D(x, t), g(x, t), \mu_{0}(t)$ and $B_{0}(t)$ are all non-negative. This functional FPE is a special case of what Basse et al. $[3,4]$ proposed for modelling the cell growth in plankton and human tumours under the assumption that the cells undergo growth, fission and mortality at given rates. It is found that in Lo's model of tumour cell growth the density function exhibits an interesting 'multipeak' structure generated by cell fission as time evolves. Within this framework the action of therapy is also examined by simply incorporating a therapy term into the deterministic cell growth term.

In all these studies the stochastic process is unbounded and the stochastic variable $x$ has a semi-infinite range $[0, \infty)$. Thus, the tumour cells could grow without limit. This is due to the fact that in the above stochastic Gompertz model the random fluctuations in the intrinsic growth rate are assumed to be independent of the size of the tumour cells. In this communication we modify the assumption in Equation (2) by introducing size-dependent random fluctuations to the intrinsic growth rate as follows:

$$
\theta(x, t)=A_{1}+\sigma \sqrt{-\ln x} \varepsilon(t) .
$$

The corresponding stochastic differential equation is then given by

$$
\mathrm{d} x=\left\{A_{1} x-A_{2} x \ln x\right\} \mathrm{d} t+\sigma x \sqrt{-\ln x} \mathrm{~d} z_{t}
$$


and represents a bounded stochastic process, i.e. the stochastic variable $x$ stays within a finite interval $I \equiv[0,1] .^{2}$ Similarly, by Ito's lemma it can be shown that the exponent $\psi \equiv-\ln x$ obeys the square-root process [7]:

$$
\mathrm{d} \psi=\left\{-A_{1}-\left(A_{2}-\frac{1}{2} \sigma^{2}\right) \psi\right\} \mathrm{d} t+\sigma \sqrt{\psi} \mathrm{d} z_{t}
$$

The constraint on the range of the stochastic variable $x$ suggests that the cells do not grow forever in size; in other words, there exists an upper bound on the size of the cells. This bounded stochastic process, which was first presented by Pesz [16] and then further generalized by Cardeal et al. [6] and Silva et al. [21], has found applications in various systems with spatial confinement, e.g. a Brownian walker trapped between fixed plates (see Ref. [14] and references therein). Hence, we shall thereafter focus on the functional FPE characterized by the following specification of the time-dependent variable coefficients: $D(x, t)=-D_{0} x^{2} \ln x, g(x, t)=\left[g_{0}-g_{1}(t) \ln x\right] x, B(x, t)=B_{0}(t) \delta(x-l)$ and $\mu(x, t)=\mu_{0}(t)$, and the absorbing boundary conditions at $x=0$ and $x=1{ }^{3}$ Undoubtedly, this new constraint on the size of tumour cells would escalate the complexity of the problem dramatically, and thus the systems are expected to exhibit more interesting properties.

As is well known, while the stationary solution of the conventional FPE can be given in closed form (at least up to quadratures) if the condition of detailed balance holds, the study of its time-dependent solution is a much more complicated problem. This situation gives rise to many stimulating opportunities for the development of approximate methods to analyse such problems. Beyond question, the 'action at a distance' effect would make the functional FPE much more challenging to treat (both analytically and numerically). Here we apply the Lie-algebraic method to derive the exact analytical solution of the functional FPE associated with the bounded stochastic process via an iterative approach and examine its properties. Obviously, the knowledge of the exact analytical solution not only provides a conceptual basis for understanding the dynamics behind the functional FPE, but it can also be useful as a benchmark to test approximate numerical or analytical procedures.

To begin with, we propose a change of variables $y=\sqrt{-\ln (x)}$ and rewrite the functional FPE in Equation (4) as follows:

$$
\begin{aligned}
\frac{\partial u(y, t)}{\partial t}= & \frac{1}{4} D_{0} \frac{\partial^{2} u(y, t)}{\partial y^{2}}+\frac{1}{2}\left\{\xi(t) y-\frac{\nu}{y}\right\} \frac{\partial u(y, t)}{\partial y}+\frac{1}{2}\left\{\xi(t)+\frac{\nu}{y^{2}}\right\} u(y, t) \\
& -\mu_{0}(t) u(y, t)+\alpha B_{0}(t) \delta(y-\sqrt{-\ln }(l / \alpha)) u(\sqrt{-\ln (l)}, t) \\
& -B_{0}(t) \delta(y-\sqrt{-\ln (l)}) u(\sqrt{-\ln (l)}, t)
\end{aligned}
$$

for $\quad 0<y<\infty, \quad$ where $\quad u(y, t)=2 y \exp \left(-y^{2}\right) P(x, t), \quad \xi(t)=g_{1}(t)-D_{0} \quad$ and $\nu=-\left[g_{0}+D_{0} / 2\right]$. This equation represents a generalization of the functional FPE associated with the well-known Rayleigh process [11]. In the absence of cell fission (i.e. $B_{0}(t)=0$ ), it is not difficult to show that Equation (7) can be recast in the following form:

$$
\frac{\partial u(y, t)}{\partial t}=\left\{a_{1}(t) \hat{K}_{+}+a_{2}(t) \hat{K}_{0}+a_{3}(t) \hat{K}_{-}+b(t)\right\} u(y, t)
$$


where

$$
\begin{gathered}
\hat{K}_{-}=\frac{1}{2}\left\{\frac{\partial^{2}}{\partial y^{2}}-\frac{2 \nu}{D_{0} y} \frac{\partial}{\partial y}+\frac{2 \nu}{D_{0} y^{2}}\right\}, \quad \hat{K}_{0}=\frac{1}{2}\left\{y \frac{\partial}{\partial y}-\frac{2 \nu-D_{0}}{2 D_{0}}\right\}, \quad \hat{K}_{+}=\frac{1}{2} y^{2}, \\
a_{3}(t)=\frac{D_{0}}{2}, \quad a_{2}(t)=\xi(t), \quad a_{1}(t)=0, \quad b(t)=\frac{1}{4}\left(1+\frac{2 \nu}{D_{0}}\right) \xi(t)-\mu_{0}(t) .
\end{gathered}
$$

The operators $\hat{K}_{+}, \hat{K}_{0}$ and $\hat{K}_{-}$are the generators of the Lie algebra su(1,1) [24]:

$$
\left[\hat{K}_{+}, \hat{K}_{-}\right]=-2 \hat{K}_{0}, \quad\left[\hat{K}_{0}, \hat{K}_{ \pm}\right]= \pm \hat{K}_{ \pm}
$$

We may define the evolution operator $\hat{U}\left(t, t_{0}\right)$ such that

$$
u(y, t)=\exp \left[\int_{t_{0}}^{t} \mathrm{~d} t^{\prime} b\left(t^{\prime}\right)\right] \cdot \hat{U}\left(t, t_{0}\right) u\left(y, t_{0}\right)
$$

Inserting Equation (11) into Equation (8) yields the evolution equation

$$
\frac{\partial}{\partial t} \hat{U}\left(t, t_{0}\right)=\left\{a_{1}(t) \hat{K}_{+}+a_{2}(t) \hat{K}_{0}+a_{3}(t) \hat{K}_{-}\right\} \hat{U}\left(t, t_{0}\right), \quad \hat{U}\left(t_{0}, t_{0}\right)=1 .
$$

Since the su(1,1) algebra is a real 'split 3-dimensional' simple Lie algebra, the WeiNorman theorem states that the evolution operator $\hat{U}\left(t, t_{0}\right)$ can be expressed in the form [23]

$$
\hat{U}\left(t, t_{0}\right)=\exp \left\{c_{1}\left(t ; t_{0}\right) \hat{K}_{+}\right\} \cdot \exp \left\{c_{2}\left(t ; t_{0}\right) \hat{K}_{0}\right\} \cdot \exp \left\{c_{3}\left(t ; t_{0}\right) \hat{K}_{-}\right\}
$$

where the coefficients $c_{i}\left(t ; t_{0}\right)$ are found to be given by (see the Appendix)

$$
c_{1}\left(t ; t_{0}\right)=0, \quad c_{2}\left(t ; t_{0}\right)=\int_{t_{0}}^{t} \xi\left(t^{\prime}\right) \mathrm{d} t^{\prime}, \quad c_{3}\left(t ; t_{0}\right)=\frac{1}{2} D_{0} \int_{t_{0}}^{t} \exp \left[c_{2}\left(t^{\prime} ; t_{0}\right)\right] \mathrm{d} t^{\prime}
$$

Without loss of generality, we suppose that $u\left(y, t_{0}\right)=y^{(\beta+1) / 2} f\left(y, t_{0}\right)$, where and $f\left(y, t_{0}\right)$ is defined in terms of the Fourier-Bessel integral:

$$
f\left(y, t_{0}\right)=\int_{0}^{\infty} \mathrm{d} \zeta \zeta J_{(\beta-1) / 2}(y \zeta) \int_{0}^{\infty} \mathrm{d} y^{\prime} y^{\prime} J_{(\beta-1) / 2}\left(y^{\prime} \zeta\right) f\left(y^{\prime}, t_{0}\right),
$$

for $\beta>1$. Then it is not difficult to show that $u(y, t)$ is given by

$$
u(y, t)=\int_{0}^{\infty} \mathrm{d} y^{\prime} G\left(y, t ; y^{\prime}, t_{0}\right) u\left(y^{\prime}, t_{0}\right)
$$


where

$$
\begin{aligned}
G\left(y, t ; y^{\prime}, t_{0}\right)= & \gamma\left(t ; t_{0}\right)\left\{y^{\prime}(1-\beta) y^{(1+\beta)} \exp \left[\frac{\beta+3}{2} c_{2}\left(t ; t_{0}\right)\right]\right\} \\
& \times \int_{0}^{\infty} \mathrm{d} \zeta \zeta \exp \left[-\frac{c_{3}\left(t ; t_{0}\right)}{2} \zeta^{2}\right] J_{(\beta-1) / 2}\left(y^{\prime} \zeta\right) \times J_{(\beta-1) / 2}\left(y \zeta \exp \left[c_{2}\left(t ; t_{0}\right) / 2\right]\right) \\
\gamma\left(t ; t_{0}\right)=\exp \{ & \left.-\int_{t_{0}}^{t} \mathrm{~d} t^{\prime} \mu_{0}\left(t^{\prime}\right)\right\} .
\end{aligned}
$$

The function $J_{p}$ is the Bessel function of the first kind of order $p$. Here we have made use of the fact that $y^{(\beta+1) / 2} J_{(\beta-1) / 2}(y \zeta)$ is an eigenfunction of the operator with the eigenvalue $-\zeta^{2} / 2$ as well as the well-known relation

$$
\exp \left(\eta y \frac{\partial}{\partial y}\right) g(y)=g(y \exp (\eta))
$$

The integral over $\zeta$ can be evaluated to give [13]

$$
\frac{1}{c_{3}\left(t ; t_{0}\right)} \exp \left\{-\frac{y^{\prime} 2-y^{2} \exp \left[c_{2}\left(t ; t_{0}\right)\right]}{2 c_{3}\left(t ; t_{0}\right)}\right\} I_{(\beta-1) / 2}\left(\frac{y^{\prime} y \exp \left\{c_{2}\left(t ; t_{0}\right) / 2\right\}}{c_{3}\left(t ; t_{0}\right)}\right)
$$

for $(\beta-1) / 2>-1, y^{\prime}>0$ and $y \exp \left[c_{2}\left(t ; t_{0}\right) / 2\right]>0$. The function $I_{p}$ is the modified Bessel function of the first kind of order $p$. The desired propagator $G\left(y, t ; y^{\prime}, t_{0}\right)$ is thus found to be

$$
\begin{aligned}
G\left(y, t ; y^{\prime}, t_{0}\right)= & \frac{\gamma\left(t ; t_{0}\right)}{c_{3}\left(t ; t_{0}\right)}\left\{y^{\prime}(1-\beta) y^{(1+\beta)} \exp \left[\frac{\beta+3}{2} c_{2}\left(t ; t_{0}\right)\right]\right\} \\
& \times \exp \left\{-\frac{y^{\prime} 2-y^{2} \exp \left[c_{2}\left(t ; t_{0}\right)\right]}{2 c_{3}\left(t ; t_{0}\right)}\right\} \times I_{(\beta-1) / 2}\left(\frac{y^{\prime} y \exp \left\{c_{2}\left(t ; t_{0}\right) / 2\right\}}{c_{3}\left(t ; t_{0}\right)}\right) .
\end{aligned}
$$

Consequently, provided that $u(y, 0)=\delta\left(y-y_{0}\right)$, the time evolution of the stochastic system is described by the propagator $G\left(y, t ; y_{0}, 0\right)$.

Next, it is straightforward to show that, in terms of the propagator $G\left(y, t ; y^{\prime}, t_{0}\right)$, the solution of the functional FPE in Equation (7) for $B_{0}(t)>0$ can be expressed as a generalized Volterra integral equation of the second kind:

$$
\begin{aligned}
u(y, t)= & \int_{0}^{\infty} \mathrm{d} y^{\prime} G\left(y, t ; y^{\prime}, 0\right) u\left(y^{\prime}, 0\right)+\int_{0}^{t} \mathrm{~d} t^{\prime} B_{0}\left(t^{\prime}\right) \\
& \times\left\{\alpha G\left(y, t ; \sqrt{-\ln (l / \alpha)}, t^{\prime}\right)-G\left(y, t ; \sqrt{-\ln (l)}, t^{\prime}\right)\right\} \times u\left(\sqrt{-\ln (l)}, t^{\prime}\right),
\end{aligned}
$$

which can be solved by the standard iterative approach:

- Initial guess:

$$
u^{(0)}(\sqrt{-\ln (l)}, t)=\int_{0}^{\infty} \mathrm{d} y^{\prime} G\left(\sqrt{-\ln (l)}, t ; y^{\prime}, 0\right) u\left(y^{\prime}, 0\right) .
$$


- Nth iteration:

$$
\begin{aligned}
u^{(N)}(\sqrt{-\ln (l)}, t)= & \int_{0}^{\infty} \mathrm{d} y^{\prime} G\left(\sqrt{-\ln (l)}, t ; y^{\prime}, 0\right) u\left(y^{\prime}, 0\right)+\int_{0}^{t} \mathrm{~d} t^{\prime} B_{0}\left(t^{\prime}\right) \\
& \times\left\{\alpha G\left(\sqrt{-\ln (l)}, t ; \sqrt{-\ln (l / \alpha)}, t^{\prime}\right)-G\left(\sqrt{-\ln (l)}, t ; \sqrt{-\ln (l)}, t^{\prime}\right)\right\} \\
& \times u^{(N-1)}\left(\sqrt{-\ln (l)}, t^{\prime}\right) \text { for } N=1,2,3, \ldots
\end{aligned}
$$

- Converged solution:

$$
\begin{aligned}
u(y, t)= & \int_{0}^{\infty} \mathrm{d} y^{\prime} G\left(y, t ; y^{\prime}, 0\right) u\left(y^{\prime}, 0\right)+\int_{0}^{t} \mathrm{~d} t^{\prime} B_{0}\left(t^{\prime}\right) \\
& \times\left\{\alpha G\left(y, t ; \sqrt{-\ln (l / \alpha)}, t^{\prime}\right)-G\left(y, t ; \sqrt{-\ln (l)}, t^{\prime}\right)\right\} \\
& \times u^{(N)}\left(\sqrt{-\ln (l)}, t^{\prime}\right) .
\end{aligned}
$$

If cell fission does not occur frequently, i.e. $B_{0}(t)$ is small, then the converged solution can be obtained after a few iterations. Alternatively, we could also express the solution $u(y, t)$ in powers of $B_{0}(t)$ as follows:

$$
\begin{aligned}
u(y, t)= & \int_{0}^{\infty} \mathrm{d} y^{\prime} u\left(y^{\prime}, 0\right) G\left(y, t ; y^{\prime}, 0\right)+\int_{0}^{\infty} \mathrm{d} y^{\prime} u\left(y^{\prime}, 0\right) \int_{0}^{t} \mathrm{~d} t^{\prime} B_{0}\left(t^{\prime}\right)\left[\alpha G\left(y, t ; \sqrt{-\ln (l / \alpha)}, t^{\prime}\right)\right. \\
& \left.-G\left(y, t ; \sqrt{-\ln (l)}, t^{\prime}\right)\right] G\left(\sqrt{-\ln (l)}, t^{\prime} ; y^{\prime}, 0\right)+\int_{0}^{\infty} \mathrm{d} y^{\prime} u\left(y^{\prime}, 0\right) \int_{0}^{t} \mathrm{~d} t^{\prime} \int_{0}^{t^{\prime}} \mathrm{d} t^{\prime \prime} B_{0}\left(t^{\prime}\right) B_{0}\left(t^{\prime \prime}\right) \\
& \times\left[\alpha G\left(y, t ; \sqrt{-\ln (l / \alpha)}, t^{\prime}\right)-G\left(y, t ; \sqrt{-\ln (l)}, t^{\prime}\right)\right] \\
& \times\left[\alpha G\left(\sqrt{-\ln (l)}, t^{\prime} ; \sqrt{-\ln (l / \alpha)}, t^{\prime \prime}\right)-G\left(\sqrt{-\ln (l)}, t^{\prime} ; \sqrt{-\ln (l)}, t^{\prime \prime}\right)\right] \\
& \times G\left(\sqrt{-\ln (l)}, t^{\prime \prime} ; y^{\prime}, 0\right)+\cdots
\end{aligned}
$$

For illustration, in Figure 1 we plot the density function $u, y, t$ versus $y$ for different values of $B_{0}(t)$ and different time $t$, provided $u(y, 0)=\delta\left(y-y_{0}\right)$. The input model parameters are selected as follows: $l=\mathrm{e}^{-1}, \alpha=2, D_{0}=1, g_{0}=1.5, g_{1}(t)=1, \mu_{0}(t)=$ 0.1 and $y_{0}=1.2$. In the absence of cell fission, i.e. $B_{0}(t)=0$, the stochastic system evolves in accordance to the Rayleigh process, and the initial density function spreads out steadily toward both endpoints due to dispersion. As we switch on the cell fission, i.e. $B_{0}(t)>0$, the qualitative features of the time evolution of the density function does not change significantly, but the cell fission induces an overall increase in the magnitude of the density function. The enhancement becomes more prominent as the rate of cell fission increases. Moreover, the density function develops a kink near $y=\sqrt{1+\ln (\alpha)}$, which corresponds to the size of the daughter cells. As shown in the figure, the kink becomes more conspicuous with the rate of cell fission and time. Finally, it should be noted that all these results are 

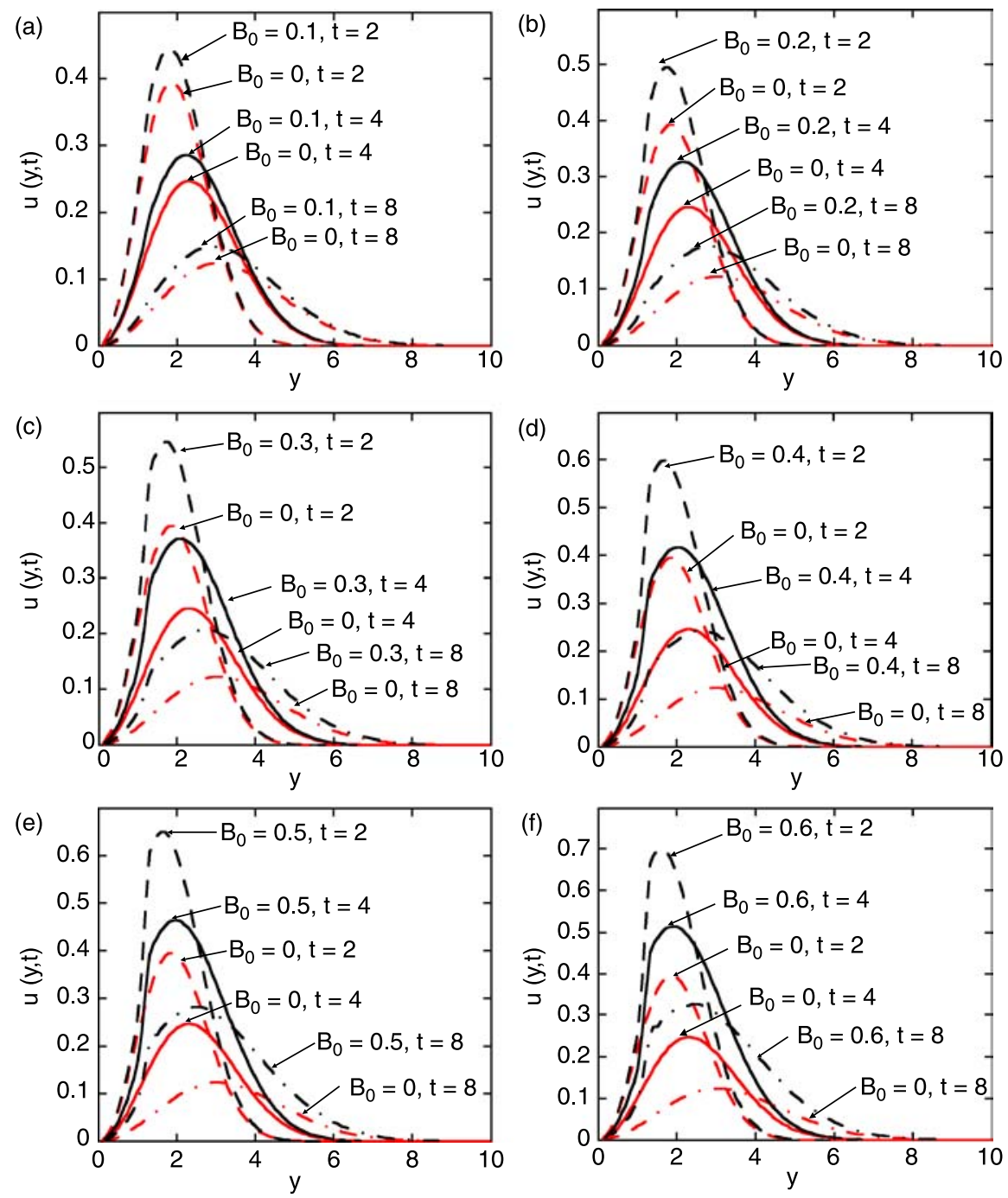

Figure 1. (a) $u(y, t)$ versus $y$ for $B_{0}(t)=0.1$ at time $t=2,4$ and 8 . (b) $u(y, t)$ versus $y$ for $B_{0}(t)=0.2$ at time $t=2,4$ and 8. (c) $u(y, t)$ versus $y$ for $B_{0}(t)=0.3$ at time $t=2,4$ and 8 . (d) $u(y, t)$ versus $y$ for $B_{0}(t)=0.4$ at time $t=2,4$ and 8 . (e) $u(y, t)$ versus $y$ for $B_{0}(t)=0.5$ at time $t=2,4$ and 8. (f) $u(y, t)$ versus $y$ for $B_{0}(t)=0.6$ at time $t=2,4$ and 8 . The input model parameters are: $l=e^{-1}$, $\alpha=2, D_{0}=1, g_{0}=1.5, g_{1}(t)=1, \mu_{0}(t)=0.1$ and $y_{0}=1.2$. For comparison, we also plot the results for the case $B_{0}(t)=0$.

obtained by evaluating the converged solution in Equation (25) up to third order of $B_{0}(t)$, with the tolerance of error being less than $1.5 \%$.

In summary, based upon the deterministic Gompertz law of cell growth, we have proposed a stochastic model of tumour cell growth, in which the size of the tumour cells is bounded, i.e. there exists an upper bound on the size of the cells. The model takes account of both cell fission (which is an 'action at a distance' effect) and mortality too. Accordingly, the density function $P(x, t)$ of the size of the tumour cells obeys a functional FPE which can be solved analytically. Beyond question, a bound on the stochastic process of the size of the tumour cells can have a large effect on the density function $P(x, t)$ and 
escalate the complexity of the problem dramatically. Furthermore, within this framework we are also able to examine the action of therapy by simply incorporating a therapy term $-W(t) x \ln x$ into the cell growth term $g(x, t)$. The additional term $W(t)$ must be nonnegative definite in order to model the elimination of a portion of tumour cells. We are now undergoing a thorough investigation in this direction, and the results will be reported elsewhere in the near future.

\section{Notes}

1. The functional FPE is a modification of the conventional FPE when the 'action at a distance' effect (i.e. cell fission in this case) is present.

2. It should be pointed out that without loss of generality we have normalized the upper bound of $x$ to unity for simplicity.

3. Following Basse et al. [3,4], in this model cell division is assumed to occur only at one critical size $x=l$ instead of over a specified size interval in order that the solution is analytically tractable.

\section{References}

[1] G. Albano and V. Giorno, A stochastic model in tumor growth, J. Theor. Biol. 242 (2006), p. 329.

[2] L. Bass, H.S. Green and H. Boxenbaum, Gompertzian mortality derived from competition between cell-types: congenial, toxicologic and biometric determinants of longevity, J. Theor. Biol. 140 (1989), p. 263.

[3] B. Basse, G.C. Wake, D.J.N. Wall, and B. van Brunt, On a cell-growth model for plankton, IMA. J. Math. Med. Biol. 21 (2004a), p. 49.

[4] B. Basse, B.C. Baguley, E.S. Marshall, G.C. Wake, and D.J.N. Wall, Modelling cell population growth with applications to cancer therapy in human tumour cell lines, Prog. Biophys. Mol. Biol. 85 (2004b), p. 353.

[5] I.D. Bassukas, Comparative Gompertzian analysis of alterations of tumor-growth patterns, Cancer Res. 54 (1994), p. 4385.

[6] J.A. Cardeal, M. de Montigny, F.C. Khanna, T.M.R. Filho, and A.E. Santana, Galilei-invariant gauge symmetries in Fokker-Planck dynamics with logarithmic diffusion and drift terms, J. Phys. A: Math. Theor. 40 (2007), p. 13467.

[7] J.C. Cox and S.A. Ross, Valuation of options for alternative stochastic processes, J. Financial Econ. 3 (1976), p. 145.

[8] L. Ferrante, S. Bompadre, L. Possati, and L. Leone, Parameter estimation in a Gompertzian stochastic model for tumor growth, Biometrics 56 (2000), p. 1076.

[9] S.C. Ferreira Jr., M.L. Matrins, and M.J. Vilela, Morphology transitions induced by chemotherapy in carcinomas in situ, Phys. Rev. E 67 (2003), p. 051914.

[10] P. Fuchshuber, M. Günther, W. Feaux de Lacroix, and R. Fischer, A mathematical model for metastatic growth illustrated by invivo and invitro growth of a transplantable mammarycarcinoma in mice, Anticancer Res. 6 (1986), p. 819.

[11] C.W. Gardiner, Handbook of Stochastic Methods for Physics, Chemistry and the Natural Sciences, 2nd ed., Springer-Verlag, Berlin, 1985.

[12] C. Guiot, P.G. Degiorgis, P.P. Delsanto, P. Gabriele, and T.S. Deisboeck, Does tumor growth follow a universal law?, J. Theor. Biol. 225 (2003), p. 147.

[13] L.S. Gradshteyn and I.M. Ryzhik, Tables of Integrals Series and Products, 5th ed., Academic Press, UK, 1994.

[14] C.F. Lo, Exact propagator of the Fokker-Planck equation with logarithmic factors in diffusion and drift terms, Phys. Lett. 319 (2003), p. 110.

[15] _ Stochastic Gompertz model of tumour cell growth, J. Theor. Biol. 248 (2007), p. 317.

[16] K. Pesz, A class of Fokker-Planck equations with logarithmic factors in diffusion and drift terms, J. Phys. A: Math. Gen. 35 (2002), p. 1827.

[17] L. Preziosi ed., Cancer Modelling and Simulation, Chapman \& Hall, London, 2003. 
[18] A.S. Qi, X. Zheng, C.Y. Du, and B.S. An, A cellular automaton model of cancerous growth, J. Theor. Biol. 161 (1993), p. 1.

[19] B. Ribba, T. Colin, and S. Schnell, A multiscale mathematical model of cancer, and its use in analyzing irradiation therapies, Theor. Biol. Med. Model. 3 (2006), p. 7.

[20] K. Rygaard and M. Spang-Thomsen, Quantitation and Gompertzian analysis of tumor growth, Breast Cancer: Res. Treat. 46 (1997), p. 303.

[21] E.M. Silva, T.M.R. Filho, and A.E. Santana, Lie symmetries of Fokker-Planck equations with logarithmic diffusion and drift terms, J. Phys.: Conference Series 40 (2006), p. 150.

[22] N.Y. Tyurin, A.Y. Yakovlev, J. Shi, and L. Bass, Testing a model of aging in animal experiments, Biometrics 51 (1995), p. 363.

[23] J. Wei and E. Norman, Lie algebraic solution of linear differential equations, J. Math. Phys. 4 (1963), p. 575.

[24] B.G. Wybourne, Classical Groups for Physicists, Wiley, New York, 1974.

\section{A Appendix: Derivation of Equation (14)}

Differentiating $U\left(t, t_{0}\right)$ in Equation (13) with respect to time $t$, we obtain

$$
\frac{\partial}{\partial t} U\left(t, t_{0}\right)=\left[h_{+}(t) K_{+}+h_{0}(t) K_{0}+h_{-}(t) K_{-}\right] U\left(t, t_{0}\right)
$$

with

$$
\begin{aligned}
& h_{+}(t)=\frac{\mathrm{d} c_{1}}{\mathrm{~d} t}-c_{1} \frac{\mathrm{d} c_{2}}{\mathrm{~d} t}+c_{1}^{2} \exp \left(-c_{2}\right) \frac{\mathrm{d} c_{3}}{\mathrm{~d} t}, \quad h_{0}(t)=\frac{\mathrm{d} c_{2}}{\mathrm{~d} t}-2 c_{1} \exp \left(-c_{2}\right) \frac{\mathrm{d} c_{3}}{\mathrm{~d} t}, \\
& h_{-}(t)=\exp \left(-c_{2}\right) \frac{\mathrm{d} c_{3}}{\mathrm{~d} t} .
\end{aligned}
$$

Then, substituting Equations (A.1) and (A.2) into Equation (12), and comparing the two sides, we have after simplication

$$
\begin{gathered}
\frac{\mathrm{d} c_{1}\left(t ; t_{0}\right)}{\mathrm{d} t}=a_{3}(t) c_{1}^{2}\left(t ; t_{0}\right)+a_{2}(t) c_{1}\left(t ; t_{0}\right), \quad c_{1}\left(t ; t_{0}\right)=0 \\
c_{2}\left(t ; t_{0}\right)=\int_{t_{0}}^{t}\left[2 a_{3}\left(t^{\prime}\right) c_{1}\left(t^{\prime} ; t_{0}\right)+a_{2}\left(t^{\prime}\right)\right] \mathrm{d} t^{\prime} \\
c_{3}\left(t ; t_{0}\right)=\int_{t_{0}}^{t} a_{3}\left(t^{\prime}\right) \exp \left[c_{2}\left(t^{\prime} ; t_{0}\right)\right] \mathrm{d} t^{\prime} .
\end{gathered}
$$

Equation (A.3), which is just a Bernoulli equation, is the equation we have to solve first to determine $c_{1}\left(t ; t_{0}\right)$, and obviously the only admissible solution is the trivial solution $c_{1}\left(t ; t_{0}\right)=0$ since it is the only one satisfying the initial condition $c_{1}\left(t ; t_{0}\right)=0$. Once $c_{1}\left(t ; t_{0}\right)$ is determined, $c_{2}\left(t ; t_{0}\right)$ and $c_{3}\left(t ; t_{0}\right)$ can be obtained readily by direct integration, as given in Equation (14). 


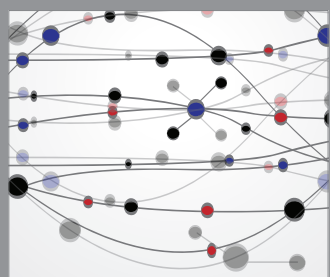

The Scientific World Journal
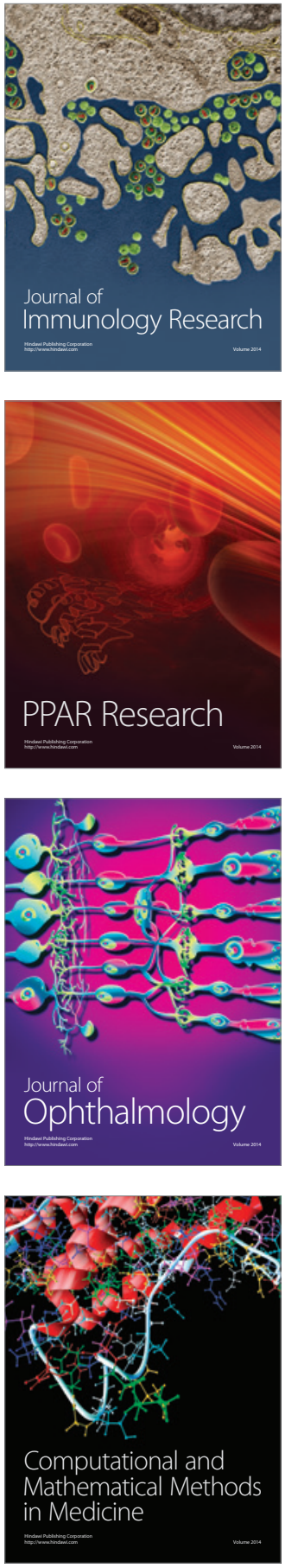

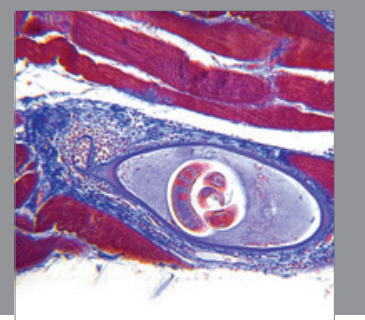

Gastroenterology

Research and Practice
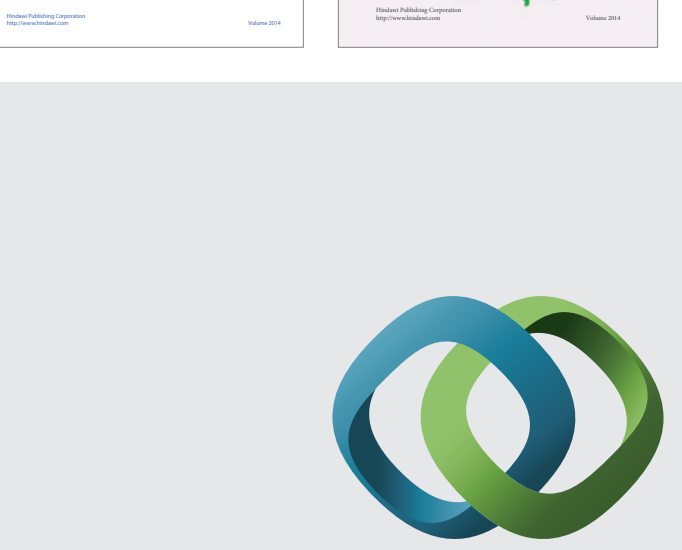

\section{Hindawi}

Submit your manuscripts at

http://www.hindawi.com
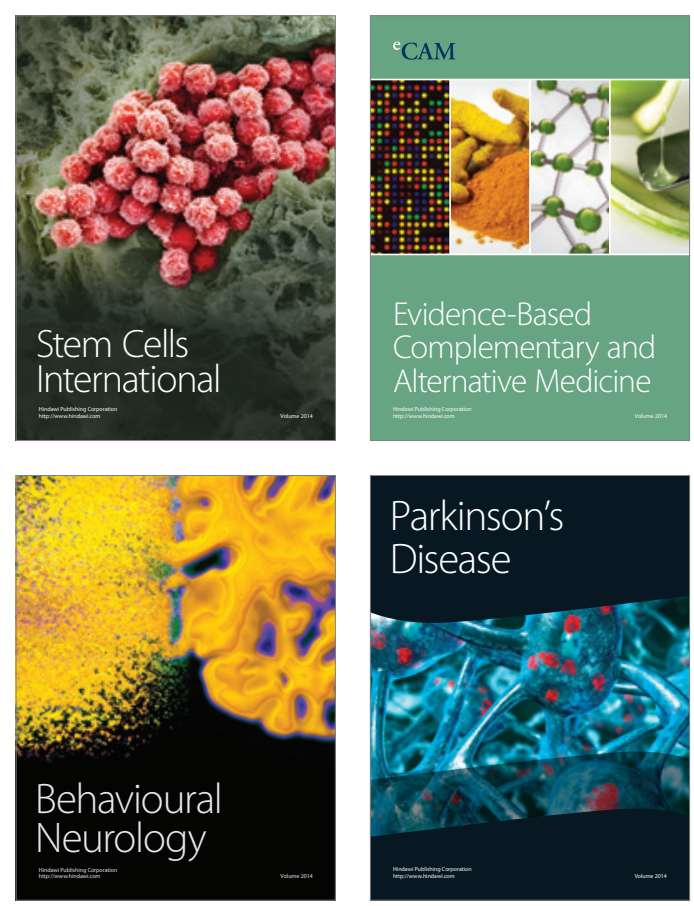

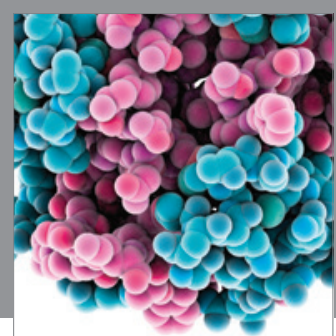

Journal of
Diabetes Research

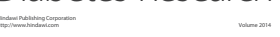

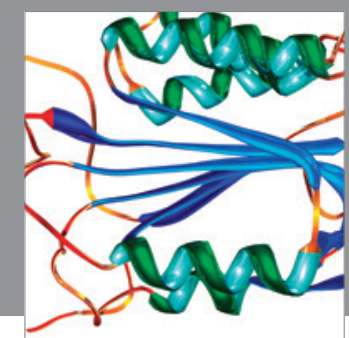

Disease Markers
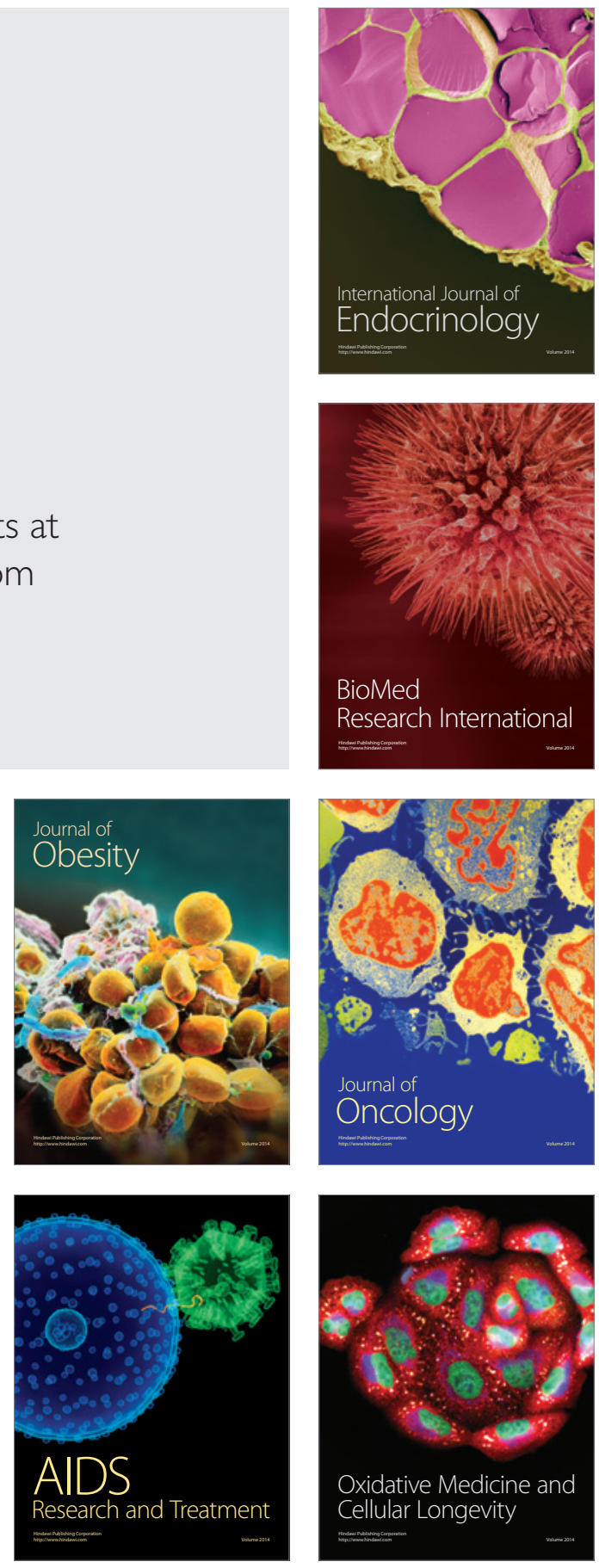